\title{
A Randomized Crossover Trial of Combination Pharmacologic Therapy in Children with Familial Hyperlipidemia
}

\author{
BRIAN W. McCRINDLE, ELIZABETH HELDEN, GERALDINE CULLEN-DEAN, AND \\ WILLIAM T. CONNER \\ Department of Pediatrics, University of Toronto, The Hospital for Sick Children, Toronto, Canada \\ [B.W.M., G.C.-D.], and Department of Pediatrics, McMaster University, St. Joseph's Hospital, Hamilton, \\ Ontario, Canada [E.H., W.T.C.]
}

\begin{abstract}
ABSTR
We sought to determine whether a low-dose combination of a
bile acid-binding resin (colestipol) with an hydroxymethylglu-
taryl CoA reductase inhibitor (pravastatin) would result in im-
proved acceptability, compliance, and effectiveness in lipid-
lowering compared with conventional therapy with a higher dose
of a bile acid-binding resin only, with fewer side effects. We
performed a randomized, crossover open-label clinical trial with
two 18 -wk medication regimens separated by an 8 -wk washout
period in 36 children and adolescents with familial hypercholes-
terolemia or familial combined hyperlipidemia. The regimens
included colestipol 10 g/d (10 pills) versus a combination of
colestipol 5 g/d with pravastatin 10 mg/d (six pills). All patients
were maintained on a fat-reduced diet. Acceptability was better
with the combination regimen. Mean compliance was similar and
suboptimal (approximately $60 \%$ with all medication compo-
\end{abstract}
nents. Mean relative LDL cholesterol lowering was significantly better with the combination regimen $(-17 \pm 16 \%$ versus $-10 \pm$ $13 \% ; p=0.045)$, although insufficient to achieve recommended target values in the majority of patients on either regimen. Both regimens were equally free of adverse effects, with no important effect on chemistry or hematologic values. Patient-reported adverse effects were more common with the conventional-dose colestipol-only regimen. Compliance with medication regimens using the bile acid-binding resins is suboptimal, although combination with a low dose of a statin may result in better lipid lowering. (Pediatr Res 51: 715-721, 2002)
Abbreviations
HMG, hydroxymethylglutaryl
NCEP, National Cholesterol Education Program

There is an extensive literature now documenting the effects of lipid-lowering pharmacologic therapy in reducing atherosclerotic cardiovascular disease and events in adults. Children with familial hyperlipidemias often have very high lipid levels, and in the great majority, fat- and cholesterol-restricted dietary therapy alone is insufficient to lower levels into the desired target range. Drug treatment with the bile acid-binding resins also does not sufficiently lower levels, and is associated with poor acceptability and compliance, especially in children (1-3). The use of the HMG CoA reductase inhibitors, or statins, is controversial in children, related to concerns regarding long-term safety, cost-effectiveness, and efficacy in terms of reducing clinical disease or events, although there are now several studies documenting effectiveness in lipid lowering and short-term safety (4-7). Given the long-term nature of therapy, compliance is one central issue. Also, there are

Received March 13, 2001; accepted January 5, 2002.

Correspondence and reprint requests: Brian McCrindle, M.D., The Hospital for Sick Children, 555 University Avenue, Toronto, Ontario, Canada M5G 1X8; e-mail: brian.mccrindle@sickkids.ca

DOI: 10.1023/01.PDR.0000015127.99522.7F no published data regarding short-term safety and effectiveness of combination drug therapy in hyperlipidemic children.

We sought to determine whether a low-dose combination of a bile acid-binding resin (colestipol) with pravastatin would result in improved acceptability, compliance, and short-term effectiveness in lipid lowering compared with conventional therapy with a higher dose of colestipol only, with fewer side effects. We also sought to determine short-term safety and effectiveness of combination drug therapy in children.

\section{METHODS}

Sample selection. Participants were recruited from the pediatric lipid disorders clinics of the Hospital for Sick Children, Toronto, and St. Joseph's Hospital, Hamilton, Ontario. All patients had been followed for a familial hyperlipidemia for at least 6 mo before consideration for recruitment. Some of these patients had participated in previous clinical trials in the clinics. Patients were either recruited by telephone contact or at the time of routinely scheduled clinic visits. All patients who were recruited met the inclusion criteria, including patient age between 8 and 
$18 \mathrm{y}$, a positive family history of hypercholesterolemia or premature atherosclerotic cardiovascular disease in first-degree relatives, a minimum fasting LDL cholesterol level before enrollment higher than $4.15 \mathrm{mM} / \mathrm{L}$, and participation and compliance in a dietary counseling program for at least 6 mo. Thus, patients were required to meet NCEP recommendations regarding need for drug therapy (8). Patients were excluded if a secondary cause was noted for their hyperlipidemia, and if there was a recent history of major surgery or serious illness within 3 mo before enrollment. Patients with extreme primary elevations of triglycerides $(>8$ $\mathrm{mM} / \mathrm{L}$ ) were not considered for this study.

Ethics. Ethics approval was obtained from the Research Ethics Boards of the Hospital for Sick Children and St. Joseph's Hospital. All parents or patients gave informed consent.

Design. This study was a randomized, open-label, crossover clinical trial. Patients were instructed to stop taking any lipidlowering medications for at least $8 \mathrm{wk}$ before the start of the study. Medication was dispensed by the clinic staff. The medication consisted of 1-g colestipol tablets and 10-mg tablets of pravastatin. Two 18-wk medication periods with an intervening 8-wk washout period constituted the study intervention. Patients were initially randomized to receive either colestipol $10 \mathrm{~g}$ per day (10 tablets) or a combination of colestipol $5 \mathrm{~g}$ per day (five tablets) together with pravastatin $10 \mathrm{mg}$ per day (one tablet). Because the aim of the study was to determine relative compliance with a low-dose combination versus conventional doses of colestipol, a pravastatin-only treatment arm was not included. Medication was provided at no cost to the study subjects. All patients were maintained on an American Heart Association step 2 diet throughout the study, with compliance assessed by a dietitian using interview and food frequency questionnaires.

Randomization procedure. Randomization was performed by one of the study investigators. Randomization was stratified by the two pediatric lipid clinics, and performed in random block sizes of four, six, and eight using a random number generator to create the assignment list. The randomization lists also specified the amount of medication to dispense, which covered two dispensing periods within each intervention, the first being $8 \mathrm{wk}$ of duration and the second being $10 \mathrm{wk}$. For each of these dispensing periods a random number of additional medication was given so that all patients would have unused medication to return.

Blinding. Study investigators and participating subjects were not blinded as to the intervention, as this was an openlabel trial. However, all laboratory assessments and data analyses were performed blinded to the intervention.

Compliance. Unused medication returned at the end of the dispensing periods was counted by the study investigators. The amount of medication taken by each patient was assumed to be the amount dispensed minus the amount returned. Compliance was expressed as the percentage of medication assumed taken versus the percentage expected to be taken if compliance was complete throughout the study period. Compliance was also assessed from a daily log book completed by the patient for the first $2 \mathrm{wk}$, and from questionnaires completed at 8 and $18 \mathrm{wk}$ after the medication period was started for each of the two regimens.

Main outcome measures. Acceptability was assessed by questionnaires at the end of the entire study, and at 8 and $18 \mathrm{wk}$ into each medication period. In addition, a final preference questionnaire was given after both medication periods were completed. Compliance was chosen as the primary outcome for the study, and was assessed by medication counts and questionnaires. Given the potential long-term nature of therapy, adequate compliance is essential before issues of effectiveness can be addressed. Effectiveness for each medication period was assessed by the change from baseline assessment to medication period end point in the fasting lipid profile. All blood samples were obtained after at least a 12-h fasting period, and assessed at each baseline and medication period end point. Profiles included assessment of total cholesterol, triglycerides, and HDL cholesterol with LDL cholesterol calculated, with assessment of apo A-1 and B-100. In addition, lipoprotein (a), homocysteine, and fibrinogen were measured at the start of study only. All assays were performed in a single standardized lipid research laboratory.

Safety monitoring. At baseline and medication period end points a complete physical examination was performed, which included assessment of height, weight, and blood pressure. Data regarding symptoms and signs were also collected in the logbooks from the first $2 \mathrm{wk}$ of each medication period and from midpoint and end-point questionnaires. Participants were instructed to contact study personnel immediately if suspected adverse effects were noted or if the patient developed serious illness, required surgery, or required other pharmacologic treatment during the medication periods. Serum chemistry studies and complete blood cell counts were assessed and compared at baseline, $2 \mathrm{wk}, 8 \mathrm{wk}$, and the end of each study medication period.

Sample size estimation. Initial sample size estimation was based on a hypothesized increase in percent compliance of 20 $\pm 25 \%$ with the combination versus the colestipol-only regimen based on paired measurements. With a desired width of the $95 \%$ confidence interval around this difference estimated at $15 \%$, approximately 40 subjects were required.

Data analysis. Data are expressed as frequencies, medians with ranges, and means with SD as appropriate. Where there is missing data, the number of nonmissing values is given. Body mass index was calculated and converted to $Z$ scores based on sex and age (9). Differences in patient and baseline characteristics at the start of the study and at the start of the second medication regimen were compared between groups based on initial randomization assignment using Fisher's exact tests, $\chi^{2}$ tests, $t$ tests, and Kruskal-Wallis ANOVA as appropriate. Differences in baseline values between the first and second regimens were compared with paired $t$ tests, with the effect of initial medication regimen assignment explored in two-way repeated measures ANOVA. Differences in compliance between the medication components were explored in repeated measures ANOVA. Paired $t$ tests were used to compare changes in outcomes measures and serum chemistry and complete blood counts with each medication regimen, with the absolute and relative effects for each medication regimen also compared with paired $t$ tests. The effect of initial medication regimen assignment was explored in two-way repeated measures ANOVA. The effect of compliance with each of the medication components on outcome measures was explored using simple linear regression analysis. All analyses were performed using SAS 
statistical software Version 7 (SAS Institute, Inc, Cary, NC, U.S.A.) using default settings. A $p<0.05$ was set as the level of statistical significance.

\section{RESULTS}

Recruitment and retention. Initially, 40 patients were enrolled from the two centers and were randomized, with 22 patients randomized to start with the combination and 18 patients randomized to start with colestipol only. However, four patients withdrew before completing the first medication period - two patients from each group. Stated reasons for each patient included the following: the family had moved, the father was ill and the family lived at a long distance from the study center, the patient was lost-to-follow-up and the family could not be located, and the patient incidentally developed hypothyroidism after baseline assessment. An additional patient starting with the colestipol only withdrew after completing the first medication period as he thought that his lipid levels were good and that he did not require medication (his LDL cholesterol level was $4.97 \mathrm{mM} / \mathrm{L}$ ). This patient's data from the first period were included in the analysis. The remaining 35 patients completed both medication periods without interruption or protocol violation. Baseline LDL cholesterol levels were above $4.9 \mathrm{mM} / \mathrm{L}$ in 28 patients $(78 \%)$, and above 4.15 $\mathrm{mM} / \mathrm{L}$ in 33 patients $(92 \%)$; thus, nearly all patients unambiguously met criteria from the NCEP Pediatric Consensus Panel for treatment with lipid-lowering medication at baseline (8). Three patients with LDL levels of 3.54, 3.73, and $3.97 \mathrm{mM} / \mathrm{L}$ at baseline were included because they had previous levels consistently above $4.15 \mathrm{mM} / \mathrm{L}$ during follow-up in the clinics.
Ten patients also had important baseline elevations of triglycerides $(>2 \mathrm{mM} / \mathrm{L})$ possibly consistent with familial combined hyperlipidemia, whereas the remainder had fasting lipid profiles and family histories consistent with the diagnosis of heterozygous familial hypercholesterolemia.

Baseline assessment. There were no significant differences between patients initially randomized to each of the two medication regimens regarding patient characteristics or baseline values, with the exception of higher apo B-100 levels in the combination regimen group (Table 1). There were also no significant differences between groups regarding baseline serum chemistry values and complete blood counts. In addition, there were no significant differences between patients at initial assessment at the start of the second or crossover medication period. To determine whether the 8-wk washout period was sufficient to return values to baseline, values at the start of each medication period were compared. Total cholesterol levels tended to be higher at the start of the first regimen (mean difference, $+0.30 \pm 0.89 \mathrm{mM} / \mathrm{L} ; p=0.054$ ), with significantly higher levels of HDL cholesterol (mean difference, $+0.10 \pm$ $0.15 \mathrm{mM} / \mathrm{L} ; p<0.001$ ) and apo A-1 (mean difference, +0.09 $\pm 0.13 \mathrm{~g} / \mathrm{L} ; p<0.001$ ). There were no significant differences in LDL cholesterol or triglyceride levels. There was no significant effect of type of initial medication regimen assignment on baseline differences between the two medication periods.

Acceptability. Acceptability as reflected by patients' preferences at the end of the study favored the combination regimen (Table 2).

Compliance. Compliance, as assessed by medication counts, was not significantly different between the high and low doses

Table 1. Characteristics at randomization

\begin{tabular}{|c|c|c|c|}
\hline \multirow[b]{2}{*}{ Variable } & \multicolumn{2}{|c|}{ Initial medication regimen assignment } & \multirow[b]{2}{*}{$p$ Value } \\
\hline & $\begin{array}{l}\text { Colestipol only* } \\
\quad(n=16)\end{array}$ & $\begin{array}{l}\text { Combination } \dagger \\
\quad(n=20)\end{array}$ & \\
\hline Sex (male:female) & $11: 5$ & $14: 6$ & 1.00 \\
\hline Median [range] age (y) & $14[10,18]$ & $14[9,18]$ & 0.88 \\
\hline Mean $( \pm \mathrm{SD})$ weight $(\mathrm{kg})$ & $63.6 \pm 18.0$ & $57.6 \pm 14.2$ & 0.27 \\
\hline Mean body mass index $\left(\mathrm{kg} / \mathrm{m}^{2}\right)$ & $24.5 \pm 5.0$ & $21.9 \pm 3.9$ & 0.09 \\
\hline Mean $Z$ score body mass index & $1.56 \pm 1.14$ & $0.83 \pm 1.31$ & 0.09 \\
\hline Mean systolic blood pressure (mm Hg) & $109 \pm 12$ & $107 \pm 10$ & 0.56 \\
\hline Mean diastolic blood pressure ( $\mathrm{mm} \mathrm{Hg}$ ) & $66 \pm 10$ & $65 \pm 7$ & 0.77 \\
\hline Previous use of lipid-lowering drugs & $9(56 \%)$ & $10 / 18(56 \%)$ & 1.00 \\
\hline \multicolumn{4}{|l|}{ Family history } \\
\hline Father with hyperlipidemia & $12 / 15(80 \%)$ & $12 / 18(67 \%)$ & 0.46 \\
\hline Father with $\mathrm{CV}$ event & $6 / 15(40 \%)$ & $6 / 18(33 \%)$ & 0.70 \\
\hline Mother with hyperlipidemia & $7 / 15(47 \%)$ & $7 / 18(39 \%)$ & 0.66 \\
\hline \multicolumn{4}{|l|}{ Mean fasting lipid profile (mM/L) } \\
\hline Total cholesterol & $7.61 \pm 1.26$ & $8.32 \pm 1.52$ & 0.15 \\
\hline LDL cholesterol & $5.91 \pm 1.20$ & $6.37 \pm 1.50$ & 0.33 \\
\hline HDL cholesterol & $1.13 \pm 0.26$ & $1.12 \pm 0.34$ & 0.96 \\
\hline Triglycerides & $1.26 \pm 0.52$ & $1.81 \pm 1.11$ & 0.08 \\
\hline Apo A-1 (g/L) & $1.14 \pm 0.17$ & $1.14 \pm 0.22$ & 0.94 \\
\hline Apo B-100 (g/L) & $1.56 \pm 0.28$ & $1.81 \pm 0.35$ & 0.03 \\
\hline Median [range] lipoprotein (a) (mg/L) & $217[22,2101]$ & $286[25,1106]$ & 0.99 \\
\hline Median [range] homocysteine $(\mu \mathrm{M} / \mathrm{L})$ & $6.3[3.6,15.1]$ & $5.8[3.1,7.2]$ & 0.12 \\
\hline Mean fibrinogen $(\mathrm{g} / \mathrm{L})$ & $2.79 \pm 0.45$ & $2.67 \pm 0.42$ & 0.85 \\
\hline
\end{tabular}

* Colestipol 10 g per day.

$\uparrow$ Colestipol $5 \mathrm{~g}$ with pravastatin $10 \mathrm{mg}$ per day.

Abbreviation: $\mathrm{CV}$, cardiovascular (myocardial infarction, stroke, angina). 
Table 2. Final preferences at end of study

\begin{tabular}{|c|c|c|c|c|}
\hline & Neither & Colestipol only* & Colestipol + pravastatin $\dagger$ & Both \\
\hline "Which form of the medicine did you think was easier to take?" & $8 \%$ & $6 \%$ & $63 \%$ & $23 \%$ \\
\hline $\begin{array}{l}\text { "Which form of the medicine do you think is best for lowering your } \\
\text { cholesterol?" }\end{array}$ & $0 \%$ & $17 \%$ & $52 \%$ & $31 \%$ \\
\hline "Which form of the medicine did you like best?" & $20 \%$ & $9 \%$ & $62 \%$ & $9 \%$ \\
\hline "Which form of the medicine would you like your doctor to give you?" & $11 \%$ & $9 \%$ & $71 \%$ & $9 \%$ \\
\hline
\end{tabular}

* Colestipol $10 \mathrm{~g}$ per day.

$\dagger$ Colestipol $5 \mathrm{~g}$ with pravastatin $10 \mathrm{mg}$ per day.

of colestipol and the pravastatin components of the regimens (Table 3). The 95\% confidence interval around the observed $2 \%$ improvement in compliance with the colestipol component of the combination versus the colestipol only regimen ranged from -20 to $+25 \%$, and therefore included the hypothesized difference of $+20 \%$, indicating that we lacked sufficient statistical power to detect this difference. Although there was a falloff in compliance between the first and second dispensing periods, this difference did not reach statistical significance. In addition, there were no significant differences in compliance between the first and second dispensing periods for each of the components of the regimens. There were no significant effects on compliance of initial medication regimen assignment.

Effectiveness. Effectiveness expressed as the absolute and relative changes in lipid profile values is shown in Table 4. With each regimen there were significant absolute and relative reductions in total and LDL cholesterol and apo B-100, with significantly greater absolute and relative reductions in total and LDL cholesterol with the combination regimen only. Although there were no significant differences in absolute or relative changes in HDL cholesterol with each medication regimen, significant increases in apo A-1 were noted, although there were no significant differences in changes between regimens. There was no significant change in triglyceride levels. Ten patients had triglyceride levels above $2 \mathrm{mM} / \mathrm{L}$ at baseline; mean absolute change in triglyceride levels for these patients while on the combination was $-0.66 \pm 0.65 \mathrm{mM} / \mathrm{L}(p=0.02)$ and on colestipol only was $+0.07 \pm 1.13 \mathrm{mM} / \mathrm{L}(p=0.86)$, and no patient had an extreme elevation of triglycerides $(>5$ $\mathrm{mM} / \mathrm{L}$ ) at any time in the study. Only three patients achieved the NCEP-recommended desired LDL cholesterol level of 2.85 $\mathrm{mM} / \mathrm{L}$ or less, one patient while on the colestipol-only regimen, and two patients while on the combination only. An additional three patients achieved the NCEP-recommended minimal LDL level of $3.35 \mathrm{mM} / \mathrm{L}$ or less, one patient while on the colestipol-only regimen and one patient while on the combination only.

Table 3. Compliance from counts of returned unused medication*

\begin{tabular}{lccc}
\hline & \multicolumn{2}{c}{ Combination } \\
\cline { 3 - 4 } & $\begin{array}{c}\text { Colestipol only } \\
(10 \mathrm{~g} / \mathrm{d})\end{array}$ & $\begin{array}{c}\text { Colestipol } \\
(5 \mathrm{~g} / \mathrm{d})\end{array}$ & $\begin{array}{c}\text { Pravastatin } \\
(10 \mathrm{mg} / \mathrm{d})\end{array}$ \\
\hline First $8 \mathrm{wk}$ & $63 \pm 29$ & $66 \pm 27$ & $65 \pm 26$ \\
Second $10 \mathrm{wk}$ & $57 \pm 44$ & $58 \pm 33$ & $60 \pm 39$ \\
Total & $60 \pm 31$ & $62 \pm 27$ & $62 \pm 28$ \\
\hline
\end{tabular}

* Expressed as a percentage of medication presumed taken $v s$ dose prescribed.
There were no significant associations between initial medication regimen assignment and changes in lipid values. The only significant association with compliance was a greater absolute LDL cholesterol lowering with better compliance with the pravastatin component of the combination regimen $(r=$ $-0.41 ; p=0.021)$.

Safety monitoring. Concerning the effects of the regimens on safety monitoring, few changes were noted. For creatine kinase, changes from baseline with both regimens were not significant at 2,8 , and $18 \mathrm{wk}$, with no significant difference noted between regimens.

Significant decreases from baseline were noted for alkaline phosphatase levels at 2 and $8 \mathrm{wk}$ for both regimens, but were only significant for the colestipol-only regimen at $18 \mathrm{wk}$. There were no significant differences in the magnitude of changes between the regimens. When compared with normal values for age and sex for our institution, no patient developed abnormally low or high levels. There were no significant changes from baseline for aspartate and alanine aminotransferase for both regimens at all time intervals. However, changes showed significant absolute reductions from baseline in the level of alanine aminotransferase for the colestipol-only relative to the combination regimen only at 8 and $18 \mathrm{wk}$. There were no other important changes relevant to any of the other chemistry or hematologic tests.

Gain in weight, height, and body mass index during each component of the study did not significantly differ between the two regimens ( $p=0.87, p=0.51, p=0.56$, respectively). Dietary compliance as assessed by food frequency questionnaire and interviews with a dietitian did not change during the study.

Adverse effects. Symptoms reported at the end of the study in the final preference questionnaire showed that the majority of patients had no symptoms, but that symptoms of constipation, bloating or gas, stomach ache, and headache were more prevalent with the colestipol-only regimen (Table 5).

\section{DISCUSSION}

Our study shows that although acceptability is better with the combination regimen, compliance was similar and suboptimal. Lipid lowering was better with the combination regimen, although insufficient to achieve NCEP recommendations in the majority on either regimen (8). Both regimens were equally safe, with no important effect on hematologic or chemistry values. Patient-reported adverse effects were more common with the higher dose colestipol-only regimen. 
Table 4. Absolute and relative changes in fasting lipid profile values

\begin{tabular}{|c|c|c|c|c|c|c|}
\hline \multirow[b]{2}{*}{ Variable } & \multicolumn{3}{|c|}{ Absolute change } & \multicolumn{3}{|c|}{ Relative change } \\
\hline & Colestipol only & Colestipol + pravastatin & $p$ & Colestipol only & Colestipol + pravastatin & $p$ \\
\hline Total cholesterol (mM/L) & $-0.63 \pm 0.80^{*}$ & $-1.06 \pm 1.11^{*}$ & 0.041 & $-7.3 \pm 10.3 \% *$ & $-12.9 \pm 12.9 \% *$ & 0.024 \\
\hline HDL cholesterol (mM/L) & $+0.01 \pm 0.18$ & $+0.03 \pm 0.13$ & 0.63 & $+2.4 \pm 19.6 \%$ & $+3.5 \pm 12.1 \%$ & 0.80 \\
\hline Triglycerides $(\mathrm{mM} / \mathrm{L})$ & $+0.11 \pm 0.68$ & $-0.07 \pm 0.72$ & 0.28 & $+11.6 \pm 45.5 \%$ & $+8.3 \pm 50.9 \%$ & 0.71 \\
\hline Apo A-1 (g/L) & $+0.06 \pm 0.16^{*}$ & $+0.07 \pm 0.13^{*}$ & 0.81 & $+7.0 \pm 18.0 \% *$ & $+7.1 \pm 12.7 \% *$ & 0.98 \\
\hline
\end{tabular}

Values are mean change $( \pm \mathrm{SD})$.

Colestipol only, $10 \mathrm{~g} / \mathrm{d}$; colestipol + pravastatin, colestipol $5 \mathrm{~g}$ with pravastatin $10 \mathrm{mg} / \mathrm{d}$.

$p$ values represent paired $t$ tests for difference between medication regimens.

$* p<0.05$ for change from start of medication regimen.

Table 5. Adverse effects

\begin{tabular}{|c|c|c|c|c|}
\hline & Neither & Colestipol only* & Colestipol + pravastatin $* *$ & Both \\
\hline $\begin{array}{l}\text { "Which form of th } \\
\text { constipation?" }\end{array}$ & $79 \%$ & $18 \%$ & $0 \%$ & $3 \%$ \\
\hline bloating/gas?" & $85 \%$ & $12 \%$ & $0 \%$ & $3 \%$ \\
\hline headache?" & $86 \%$ & $11 \%$ & $0 \%$ & $3 \%$ \\
\hline muscle ache?" & $91 \%$ & $6 \%$ & $3 \%$ & $0 \%$ \\
\hline
\end{tabular}

* Colestipol 10 g per day.

** Colestipol $5 \mathrm{~g}$ with pravastatin $10 \mathrm{mg}$ per day.

Autopsy studies in children, adolescents, and young adults have shown that the atherosclerotic process begins and progresses throughout childhood, and the extent of vascular involvement is directly related to known risk factors, particularly hyperlipidemia (10-13). Studies in adults have shown that effective lipid lowering with pharmacologic agents can reduce morbidity and mortality from atherosclerotic cardiovascular events. Patients with familial hyperlipidemias, particularly defects in the LDL receptor or its recognition apolipoprotein (familial hypercholesterolemia), are at greatly increased risk of premature cardiovascular disease, with the majority of affected males having cardiovascular events in their thirties and forties. Evidence of an effect of lipid lowering on vascular end points in children and adolescents is currently lacking. However, the development of newer noninvasive indicators of the atherosclerotic process, such as assessment of brachial artery reactivity (14-16), coronary artery calcification $(17,18)$, and increased carotid artery intima-media thickness $(16,19,20)$, may serve as potential targets for monitoring interventions in this age range.

The NCEP Consensus Panel recommended that pharmacologic therapy be considered only after an adequate trial of at least 6 to 12 mo of a fat- and cholesterol-restricted diet, and if LDL cholesterol levels remained above $4.9 \mathrm{mM} / \mathrm{L}$, or above $4.15 \mathrm{mM} / \mathrm{L}$ with either a positive family history of premature cardiovascular disease or the presence of two or more additional persistent personal risk factors (8). Ose and Tonstad (21) have proposed an algorithm that takes into account the patient's sex, family history, and magnitude of the LDL cholesterol elevation in recommending the age at which medication should be started. Specific family history factors to consider include the number of family members who have hyperlipidemia or premature cardiovascular events, earlier age and greater severity of an event, and an event in a parent or female family member. Additional factors that may influence the age at which medication may be started include the type of underlying metabolic abnormality and whether there is associated high triglycerides or low HDL cholesterol (22).

A recent national survey showed that whereas pediatricians were more likely to screen and initiate treatment for risk factors at a younger age, family and general practitioners were more likely to treat hyperlipidemia in children with drugs, particularly the statins (23). This probably represents an extrapolation based on the greater experience most family and general practitioners have in using the statins to treat hyperlipidemia in their adult patients.

Studies of lipid-lowering pharmacologic therapy specifically in children and adolescents are summarized in Table $6(1-7$, $24,25)$. The use of the bile acid-binding resins, cholestyramine and colestipol, although safe, is associated with only modest reductions in LDL cholesterol and poor acceptability and compliance (1-3). The bile acid-binding resins work by binding bile salts in the intestinal lumen and preventing their enterohepatic circulation, thereby eliminating them from the cholesterol pool. The liver cells then up-regulate the production of LDL receptors, to bring more cholesterol into the cells for synthesis of bile salts, thereby decreasing circulating LDL cholesterol levels. These agents are believed to be safe because they are not systemically absorbed. However, gastrointestinal adverse effects are common, and both acceptability and compliance are poor (1-3). We have previously shown that compliance is unrelated to patient characteristics, attitudes, and perceptions (2). Studies with the statins, specifically simvastatin (4), pravastatin (5), and lovastatin $(6,7)$, have shown good reductions in LDL cholesterol with modest elevations in HDL cholesterol. The statins work by inhibiting the rate-limiting enzyme for endogenous cholesterol synthesis, thus creating an intracellular demand for cholesterol, which is addressed by the 
Table 6. Efficacy and safety of lipid-lowering drug therapy in children and adolescents

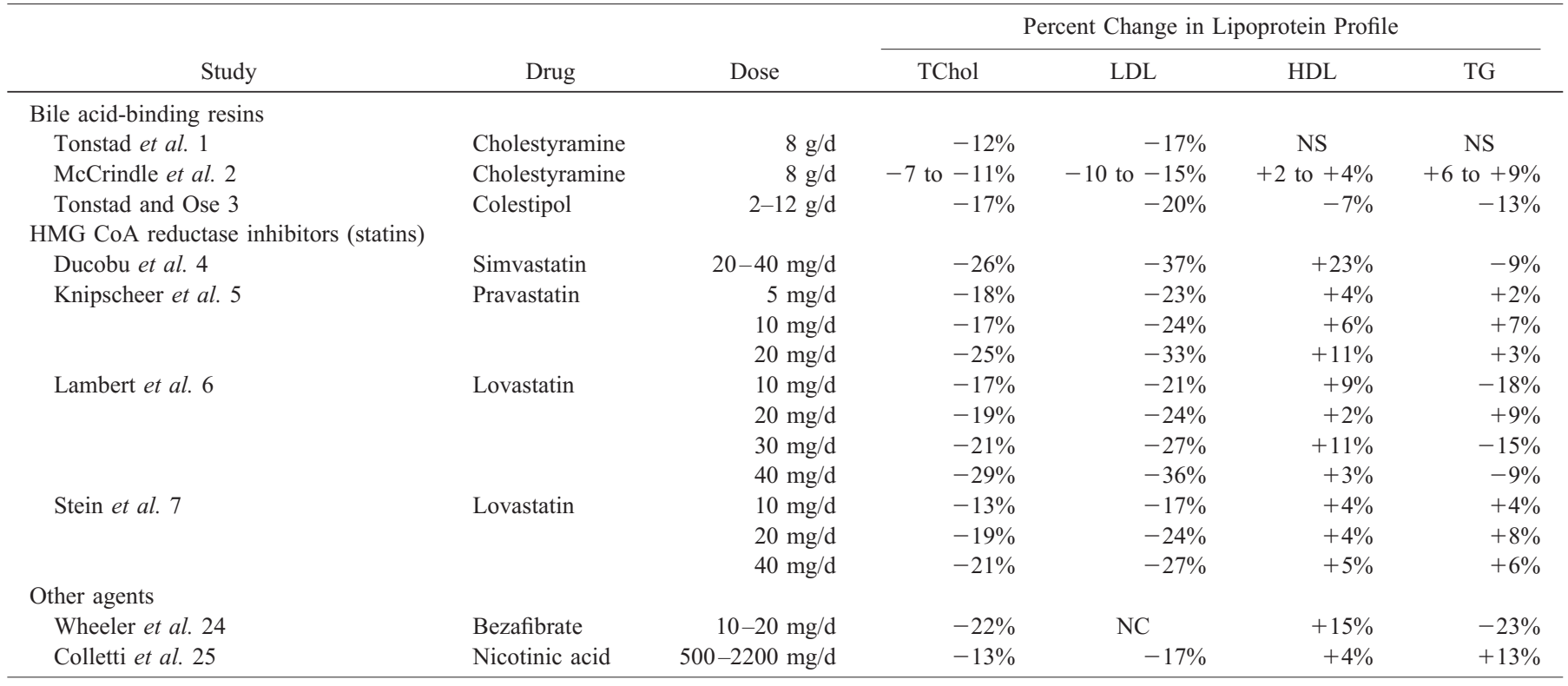

Abbreviations: NC, not calculated; NS, not significant; TChol, total cholesterol; TG, triglycerides.

up-regulation of LDL receptors with increase clearance of circulating LDL cholesterol. Adverse effects have been minimal, although these studies have been of relatively short-term duration. Experiences with other types of lipid-lowering drugs in children and adolescents have been limited, and there have been no studies of combination therapy. Although our study showed significant reductions of LDL cholesterol with the combination therapy, the vast majority of our patients had still failed to achieve recommended target levels. This is generally true of patients with familial hypercholesterolemia, who have more-extreme elevations of LDL cholesterol and, therefore, require greater percent reductions from baseline values than can be easily achieved with the bile acid-binding resins alone. Only nine of the 36 patients in our study had initial baseline LDL cholesterol levels that would be decreased to below 4.1 $\mathrm{mM} / \mathrm{L}$ if a $25 \%$ reduction could be achieved. In addition, the lesser degree of LDL lowering observed with pravastatin in our study compared with that reported by Knipscheer et al. (5) probably represents the higher initial LDL levels in our population, and the effects of diminished compliance probably attributable to the concomitant use of colestipol. Hennermann et al. (26) studied the effects of diet and drug therapy in 71 children with familial hypercholesterolemia, and although noting significant effectiveness, the vast majority continued to have important hyperlipidemia. The general recommendation of the statins awaits long-term studies, evidence of alteration of the atherosclerotic disease process, and proof of costeffectiveness in this population (27).

It is likely that solitary therapy with the statins may be the only acceptable and effective therapy for lipid lowering in high-risk children. However, the use of the statins in these age groups should be under monitored circumstances, preferably as part of a clinical study. Before widespread acceptance and use, data are needed regarding long-term safety and effectiveness, impact on vascular end points or clinical events, and evidence of cost-effectiveness.

\section{REFERENCES}

1. Tonstad S, Knudtzon J, Sivertsen M, Refsum H, Ose L 1996 Efficacy and safety of cholestyramine therapy in peripubertal and prepubertal children with familial hypercholesterolemia. J Pediatr 129:42-49

2. McCrindle BW, O'Neill MB, Cullen-Dean G, Helden E 1997 Acceptability and compliance with two forms of cholestyramine in the treatment of hypercholesterolemia in children: a randomized, crossover trial. J Pediatr 130:266-273

3. Tonstad S, Ose L 1996 Colestipol tablets in adolescents with familial hypercholesterolaemia. Acta Paediatr 85:1080-1082

4. Ducobu J, Brasseur D, Chaudron JM, Deslypere JP, Harvengt C, Muls E, Thomson M 1992 Simvastatin use in children [letter]. Lancet 339:1488

5. Knipscheer HC, Boelen CC, Kastelein JJ, van Diermen DE, Groenemeijer BE, van den Ende A, Buller HR, Bakker HD 1996 Short-term efficacy and safety of pravastatin in 72 children with familial hypercholesterolemia. Pediatr Res 39:867-871

6. Lambert M, Lupien PJ, Gagne C, Levy E, Blaichman S, Langlois S, Hayden M, Rose V, Clarke JT, Wolfe BM, Clarson C, Parsons H, Stephure DK, Potvin D, Lambert J 1996 Treatment of familial hypercholesterolemia in children and adolescents: effect of lovastatin. Canadian Lovastatin in Children Study Group. Pediatrics 97:619-628

7. Stein EA, Illingworth DR, Kwiterovich Jr PO, Liacouris CA, Siimes MA, Jacobson MS, Brewster TG, Hopkins P, Davidson M, Graham K, Arensman F, Knopp RH, DuJovne C, Williams CL, Isaacsohn JL, Jacobsen CA, Laskarzewski PM, Ames S, Gormley GJ 1999 Efficacy and safety of lovastatin in adolescent males with heterozygous familial hypercholesterolemia: a randomized controlled trial. JAMA 281:137144

8. American Academy of Pediatrics 1992 National Cholesterol Education Program: Report of the Expert Panel on Blood Cholesterol Levels in Children and Adolescents. Pediatrics 89:525-584

9. Cole TJ, Freeman JV, Preece MA 1996 Body mass index reference curves for the UK, 1990. Arch Dis Child 73:25-29

10. McGill HC, McMahan CA 1998 Determinants of atherosclerosis in the young. Pathobiological Determinants of Atherosclerosis in Youth (PDAY) Research Group. Am J Cardiol 82:30T-36T

11. Berenson GS, Wattigney WA, Tracy RE, Newman III WP, Srinivasan SR, Webber LS, Dalferes Jr ER, Strong JP 1992 Atherosclerosis of the aorta and coronary arteries and cardiovascular risk factors in persons aged 6 to 30 years and studied at necropsy (The Bogalusa Heart Study). Am J Cardiol 70:851-858

12. Berenson GS, Srinivasan SR, Bao W, Newman III WP, Tracy RE, Wattigney WA 1998 Association between multiple cardiovascular risk factors and atherosclerosis in children and young adults. The Bogalusa Heart Study. N Engl J Med 338:1650-1656

13. Strong JP, Malcom GT, McMahan CA, Tracy RE, Newman WP, Herderick EE, Cornhill JF 1999 Prevalence and extent of atherosclerosis in adolescents and young adults: implications for prevention from the Pathobiological Determinants of Atherosclerosis in Youth Study. JAMA 281:727-735

14. Celermajer DS, Sorensen KE, Gooch VM, Spiegelhalter DJ, Miller OI, Sullivan ID, Lloyd JK, Deanfield JE 1992 Non-invasive detection of endothelial dysfunction in children and adults at risk of atherosclerosis. Lancet 340:1111-1115

15. Sorensen KE, Celermajer DS, Georgakopoulos D, Hatcher G, Betteridge DJ, Deanfield JE 1994 Impairment of endothelium-dependent dilation is an early event in children with familial hypercholesterolemia and is related to the lipoprotein(a) level. J Clin Invest 93:50-55 
16. Aggoun Y, Bonnet D, Sidi D, Girardet JP, Brucker E, Polak M, Safar ME, Levy BI 2000 Arterial mechanical changes in children with familial hyperlipidemia. Arterioscler Thromb Vasc Biol 20:2070-2075

17. Mahoney LT, Burns TL, Stanford W, Thompson BH, Witt JD, Rost CA, Lauer RM 1996 Coronary risk factors measured in childhood and young adult life are associated with coronary artery calcification in young adults: the Muscatine Study. J Am Coll Cardiol 27:277-284

18. Davis PH, Dawson JD, Mahoney LT, Lauer RM 1999 Increased carotid intimalmedial thickness and coronary calcification are related in young and middle-aged adults. The Muscatine Study. Circulation 100:838-842

19. Pauciullo P, Iannuzzi A, Sartorio R, Irace C, Covetti G, Di Costanzo A, Rubba P 1994 Increased intima-media thickness of the common carotid artery in hypercholesterolemic children. Arterioscler Thromb 14:1075-1079

20. Tonstad S, Joakimsen O, Stensland-Bugge E, Leren TP, Ose L, Russell D, Bonaa KH 1996 Risk factors related to carotid intima-media thickness and plaque in children with familial hypercholesterolemia and control subjects. Arterioscler Thromb Vasc Biol 16:984-991
21. Ose L, Tonstad S 1995 The detection and management of dyslipidaemia in children and adolescents. Acta Paediatr 84:1213-1215

22. Tonstad S 2000 Role of lipid-lowering pharmacotherapy in children. Paediatr Drugs $2: 11-22$

23. Kimm SY, Payne GH, Stylianou MP, Waclawiw MA, Lichtenstein C 1998 National trends in the management of cardiovascular disease risk factors in children: second NHLBI survey of primary care physicians. Pediatrics 102:E50

24. Wheeler KA, West RJ, Lloyd JK, Barley J 1985 Double blind trial of bezafibrate in familial hypercholesterolaemia. Arch Dis Child 60:34-37

25. Colletti RB, Neufeld EJ, Roff NK, McAuliffe TL, Baker AL, Newburger JW 1993 Niacin treatment of hypercholesterolemia in children. Pediatrics 92:78-82

26. Hennermann JB, Herwig J, Marz W, Asskali F, Bohles HJ 1998 Lipid and lipoprotein profiles in children with familial hypercholesterolaemia: effects of therapy. Eur J Pediatr 157:912-918

27. Duplaga BA 1999 Treatment of childhood hypercholesterolemia with HMG-CoA reductase inhibitors. Ann Pharmacother 33:1224-1227 